\title{
Behavioral Measures in Myopic and Non-Myopic High Schoolers During the Covid-19 Pandemic
}

\author{
Divya Shukla ${ }^{1}$ and Lisa Ostrin\#
}

${ }^{1}$ Carnegie Vanguard High School, Houston, TX, USA

\#Advisor

\section{$\underline{\text { ABSTRACT }}$}

Purpose: The purpose of this study is to understand how the visual activity of highschool students in Houston changed due to quarantine and online learning during the COVID-19 pandemic, and whether these behaviors were different between myopes and non-myopes. Methods: Thirty-one participants ( $16.3 \pm 0.8$ years, 8 females), including 12 myopes and 8 non-myopes, wore a Clouclip and Actiwatch for a week. The Clouclip records working distance and the Actiwatch measures light exposure, physical activity, and sleep duration. A questionnaire was also completed regarding demographics, ocular history, and visual behaviors. Results: Data showed that myopic participants engaged in near work (working distances of 10 to $<60 \mathrm{~cm}$ ) for $8.4 \pm 2.6$ hours on a week day and $6.5 \pm 2.1$ hours on a weekend day. Nonmyopic participants engaged in near work for $6.1 \pm 2.7$ hours on a week day and $4.5 \pm 1.9$ hours on a weekend day. While weekend near work was significantly different between refractive error groups $(\mathrm{P}<.05)$, weekday near work $(\mathrm{P}=.08)$ was not. There were no differences between refractive error groups for daily light exposure $(\mathrm{P}=.89)$, time outdoors $(\mathrm{P}=.44)$, or sleep duration $(\mathrm{P}=.80)$. Conclusions: There was no significant change in sleep duration and physical activity regardless of refractive error over the pandemic, while results revealed a significant decrease in outdoor light exposure and the increase in near and intermediate work, especially with electronic devices such as the computer.

\section{Introduction}

Myopia, also known as near-sightedness, is one of the most common ophthalmic conditions affecting approximately one-third of the world's population today (Eppenberger et al., 2020), which is roughly 2.5 billion people, and its prevalence continues to grow. It was estimated that $4 \%$ of the global population was affected by high myopia in 2010 (Eppenberger et al., 2020), and by 2018, 22.9\% of the world's population, or 1.406 billion people (Theophanous et al., 2018), were affected. In recent studies, results show that prevalence rates of myopia can reach up to $80-90 \%$ among young adults in East Asia, with 20\% of them having developed high myopia (Morgan et al., 2012, Eppenberger et al., 2020). Europe indicates an estimated prevalence of $27 \%$ while studies in children have shown prevalence rates of 41.9\% in North America and 11.6\% in South America (Eppenberger et al., 2020). As myopia continues to prevail in many regions across the world, it is expected that by 2050, around half the global population (49.8\%) will be affected by myopia, potentially impacting over 4.7 billion worldwide (Holden et al., 2016, Eppenberger et al., 2020).

\section{Diopters}

Myopia is measured in diopters (D), a unit of measurement referring to refractive power. A diopter is equal to the reciprocal of the focal length of a lens. The focal length is the distance between the center of the lens and the point at which the object is brought into clear focus. For example, a focal length of one meter would be equivalent to $1 / 1$, or $1 \mathrm{D}$, and a focal length of $50 \mathrm{~cm}$ would be equivalent to $1 / 0.5$, or $2 \mathrm{D}$. For those with myopia, the refracting power of 
the eye is too strong, and a negative powered optical lens is used to bring the focal point of parallel rays entering the eye to the retina (Eppenberger et al., 2020).

\section{Definition of Myopia}

Though definitions of myopia vary across studies, myopia is most commonly defined as spherical equivalent refraction of $-0.50 \mathrm{D}$ or more minus, with up to $-3.00 \mathrm{D}$ considered to be mild myopia (Holden et al., 2016). Refractive errors of $-3.00 \mathrm{D}$ to $-6.00 \mathrm{D}$ are considered to be moderate myopia, and more negative than $-6.00 \mathrm{D}$ is most commonly defined as high myopia (Holden et al., 2016). Typically, myopia occurs because the eye grows too long. Due to this increase in axial length, light rays focus in front of the retina producing blurred images (Eppenberger et al., 2020). Additionally, this elongation leads to structural changes of several ocular structures, including the retina, choroid, and sclera. While myopia can be corrected by LASIK surgery, contact lens, or glasses, the elongation of the eye is permanent and can lead to ocular pathologies, such as retinal degeneration, retinal detachment, glaucoma, cataract, visual impairment, and blindness (Huang et al., 2015, Flitcroft 2012). Efforts have increased to understand the regulatory mechanisms underlying myopic eye growth due to the potentially blinding complications and socioeconomic burden associated with high myopia (Chia et al., 2001). Those without prescription and those who can see far away objects in sharp focus in the neutral and relaxed state are considered emmetropes, or non-myopes.

\section{Impact of the COVID-19 Pandemic}

In January of 2020, the first cases of the COVID-19 global pandemic were diagnosed in the United States (Holshue et al., 2020), and by March, schools closed face-to-face learning and transitioned to remote learning through online schooling, which has continued for many into the spring of 2021. Though there is no existing evidence that the coronavirus directly affects the biology of the eye, the transition to online schooling during the COVID pandemic dramatically changed children's lifestyles, leading to an increased number of hours spent on electronic devices and limited outdoor light exposure. High school students are particularly affected because of the heavy course loads (Gill et al., 2010) leading to significantly higher hours of near work and screen time. The effects of this transition on children's eyes are unknown (Navel et al., 2020). These behavioral changes may affect the way the children's eyes grow, and that eye growth is what causes myopia. Therefore, it is important that studies are conducted to research the behaviors associated with myopia, which will allow modifiable behavioral recommendations to be made to maintain emmetropia or mitigate myopia in children.

\section{Literature Review}

Refractive development is the outcome of complex interactions of genetic, environmental, and behavioral factors. The prevalence of myopia has been increasing faster than genetics can account for (Chia et al., 2001), suggesting that children's behaviors, such as time spent outdoors (Eppenberger et al., 2020, Read et al., 2014), near work (Chia et al., 2001, Huang et al., 2015, Navel et al., 2020), and electronic device use (Lanca et al., 2020), might be contributing to this increase. Near work refers to activities with a short working distance, generally less than $60 \mathrm{~cm}$, such as reading, writing, using hand-held electronic devices, and playing video games (Huang et al., 2015, Ostrin et al., 2018, Bhandari et al., 2020).

\section{Near Work}

Evidence regarding the influence of near work in myopia pathogenesis is conflicting, with some studies reporting no significant associations (Saw et al., 2000, Wu et al., 2015, Lu et al., 2009), and others reporting significant links 
(Huang et al., 2015, Navel et al., 2020, Chia et al., 2001, Harb et al., 2006). For example, SM Saw, from the Department of Community at the Occupational and Family Medicine from National University of Singapore, conducted a concurrent cohort study where she gathered one-hundred-fifty-three children in Singapore between six to twelve years to participate in a face-to-face clinic interview. After observing the refractions in these children for two years, Saw concluded that socio-economic status and nearwork activities were not related to myopia progression. On the other hand, researcher HM Huang, from the department of Ophthalmology at the Chang Gung Memorial Hospital and Chang Gung University College of Medicine in Taiwan, led a systematic review and metaanalysis to determine the association between near work and myopia. Through her evaluation of existing papers and statistical analysis, she concluded “individuals who performed more near work activities had an 80\% higher risk of having myopia" (Huang et al., 2015) which indicates a strong association between myopia and near work. Many studies support both the conclusions of Saw and Huang, indicating that the effect of near work on myopia is inconsistent.

\section{Outdoor Time and Light Exposure}

The literature with respect to time outdoors has been more consistent, with many studies reporting that increased time spent outdoors decreases the prevalence (Eppenberger et al., 2020, Theophanous et al., 2018, Landis et al., 2018, Read et al., 2014) and progression of myopia (Eppenberger et al., 2020, Landis et al., 2018). EG Landis, a member of the department of Ophthalmology and Neuroscience at Emory University in Georgia, conducted an experimental study to test the influence of different intensities of light on refractive error and myopia prevention. In this study, the variations of light exposure on weekends and weekdays were measured between myopes and non-myopes through wearable light sensors. After analyzing the data, Landis concluded that there were "significant differences in average daily light exposure between myopic and non-myopic children" (Landis et al., 2018). These results also indicate that in addition to bright light exposure, the rod pathways stimulated by dim light exposure may influence eye growth and myopia development. Several other studies align with Landis' findings that light exposure is associated with refractive error.

\section{Other Behavioral Factors}

Other behavioral factors, such as electronic device use and sleep, may influence myopia. Some studies indicate that the introduction of digital devices does not influence myopia or eye growth (Singh et al., 2020), whereas other studies indicate that "digital screen time has been cited as a potential modifiable environmental risk factor that can increase myopia risk" (Lanca et al., 2020). Speculation exists whether this increase in screen time, in combination with fewer hours of outdoor light exposure, may lead to increased myopia. Regarding the literature related to sleep and physical activity, studies indicate that there is an inverse relationship between sleep duration and myopia (Jee et al., 2015).

\section{Purpose}

The overall goal of this study is to understand how the visual activity of high school students in the Houston area changed due to quarantine and online learning during the COVID19 global pandemic, and whether these behaviors were different between emmetropes (those without nearsightedness) and myopes (those with nearsightedness). Investigating the behaviors of high school students who are myopic and those who are not through the use of objective continuously measuring sensors, including rangefinders, light sensors, and activity monitors, complemented with activity questionnaires, will provide insight into how children's behaviors changed during the COVID-19 pandemic and will help with understanding how these behaviors may influence eye growth. 


\section{Significance}

Ultimately, these findings will contribute to the development of modifiable behavioral recommendations to reduce myopia prevalence and minimize progression (Navel et al., 2020). If certain behaviors indicate a strong association with myopia, recommendations to minimize those particular behaviors may be provided to those with progressing myopia. Furthermore, data retrieved from this study may be of interest to other fields relating to child behavior and public health to better understand the impact of quarantine on children's behaviors.

\section{Methods}

To properly determine the association between behaviors with refractive error and the pandemic, a cross-sectional study of high school students in Houston, Texas, was performed. A cross-sectional study allows one to observe and analyze data from a specific population from a specific point in time. This is the most suitable approach compared to other methods since it will allow data to be collected and analyzed at a single time point during the pandemic from a representative subset of high school students. Environmental and behavioral factors associated with myopia in children will be quantified using a custom questionnaire and objective continuous tracking devices. These devices have been successfully used in past studies (Ostrin et al., 2018, Bhandari et al., 2020, Bélanger et al., 2013) investigating environmental and behavioral factors in children and adults. Execution of this project will require minimal interaction between study personnel and participants, which is crucial in this period of social distancing. The study was approved by the University of Houston Institutional Review Board.

\section{Hypothesis}

The central hypothesis that light exposure, near work, and electronic device use vary by refractive error group will be tested. Refractive error groups will include participants with nearsightedness and participants with unaided normal vision. An additional hypothesis that children's behaviors changed during the COVD-19 pandemic will be tested through a questionnaire. Data will be analyzed by refractive error group as well as by time period (before vs during the COVID-19 pandemic).

\section{Participants}

Houston high school students between the ages of 13-18 years were recruited by flyers for this cross-sectional study. All parents provided written consent and children provided assent. Each participant was prescreened virtually to ensure eligibility and provide an opportunity for both parents and participants to ask questions prior to enrollment. Exclusion criteria included those with previous ocular surgery, a systemic disease known to cause variability in refractive error, a history of ocular trauma, or hyperopia (farsightedness). To minimize bias that is typical with questionnaires, two objectively measuring wearable sensor devices were used to collect data on current behaviors during the pandemic. Additionally, a questionnaire was administered to collect data on current behaviors during the pandemic as well as typical behaviors before the pandemic. The data collected from the devices was compared with the data reported in the survey.

\section{Measurement of Light, Activity, and Sleep}

Following the prescreening, an actigraph device (Actiwatch Spectrum, Philips Respironics, Bend, OR) was dispensed for participants to wear continuously for a week for objective continuous measurement of light exposure, physical activity, and sleep. The Actiwatch Spectrum (Figure 1) is a lightweight and non-invasive wristwatch that monitors 
ambient light exposure and activity continuously at $32 \mathrm{~Hz}$ (Ostrin et al., 2018) and is commonly used in studies related to myopia, circadian rhythm, and sleep (Ostrin et al., 2018, Bélanger et al., 2013). The date and time are both displayed on the screen of the watch, and the device has a built-in "offwrist" detection system to monitor participant compliance. This device is equipped with three color light sensors to determine exposure to three color bands of the visible spectrum: red, green, and blue (Ostrin et al., 2018) in irradiance. The illuminance of white light is recorded in units of lux (range 0.1 to 200,000 lux), and the irradiance of the red, green, and blue spectral components are measured in $\mu \mathrm{W} / \mathrm{cm} 2$ (red channel: 400-500 nm, green channel: 500-600 nm, blue channel: 600-700 nm) (Ostrin et al., 2018). Minutes exposed to higher than 1000 lux were used as an approximation for time spent outdoors during the day (Ostrin et al., 2018).

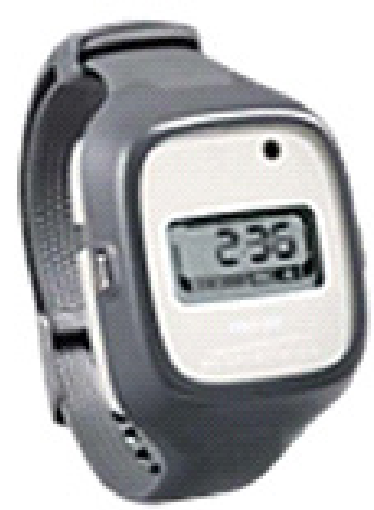

Figure 1. Actiwatch Spectrum Wristwatch; measures physical activity, light exposure, and sleep.

The device is also equipped with a highly sensitive solid-state piezoelectric accelerometer (Ostrin 2018) and tracks physical activity in "counts per minute" (cpm). The Actiwatch Spectrum Plus is waterproof up to IPX7 standards and resistant to heat, perspiration, and cold. Devices with IPX7 standards indicate that the device can be immersed in water up to 1 meter in depth for up to 30 minutes allowing the Actiwatch to be used in the shower and other waterrelated activities without damage. Actiwatch was set to average data over one-minute epochs. Over the course of the week, mean daily light exposure, time spent outdoors, physical activity, and sleep duration were determined for each participant. The device software (Actiware 6.1.1, Philips Respironics, Bend, OR) was used to retrieve and analyze the data. The Actiwatch connects to the software on the computer through a mini micro-USB cable. Once the device has been recognized, the data are retrieved, the device is put to sleep, and the device can then be configured for the following participant. The software uses algorithms to defines sleep parameters, such as duration, latency, and efficiency, while also determining active and rest time (Bélanger et al., 2013).

\section{Objective Rangefinder}

A rangefinder (Clouclip Glasses Clip M2) was also dispensed for participants to wear during wake hours continuously for a week, with the exception of water-related activities such as swimming or showering. The purpose of the Clouclip is to provide data on time spent performing near work and outdoor light exposure. This objective rangefinder measures viewing distance through an infrared sensor and eye-level illuminance through an integrated chip in the device (Wen, Bhandari 2020). The Clouclip device attaches to a pair of glasses by sliding into a small rubber sleeve mounted at the front of the right temple of the glasses (Figure 2). Participants who were emmetropic, and myopes who preferred to wear contacts, were provided with a pair of Plano glasses (i.e. nonprescription) to hold the Clouclip. Participants were instructed to charge the lithium polymer battery $(40 \mathrm{mAH})$ with a USB cable with a magnetic charging port each night. A small green light indicates full charge and a red light when plugged in indicates lack of charge. After one 
week, the data from each device were transmitted via Bluetooth to the Clouclip Medical app on a smartphone (requires Android 4.4 or later, IOS 9.0 or later) and uploaded to a server provided by the Clouclip developer.

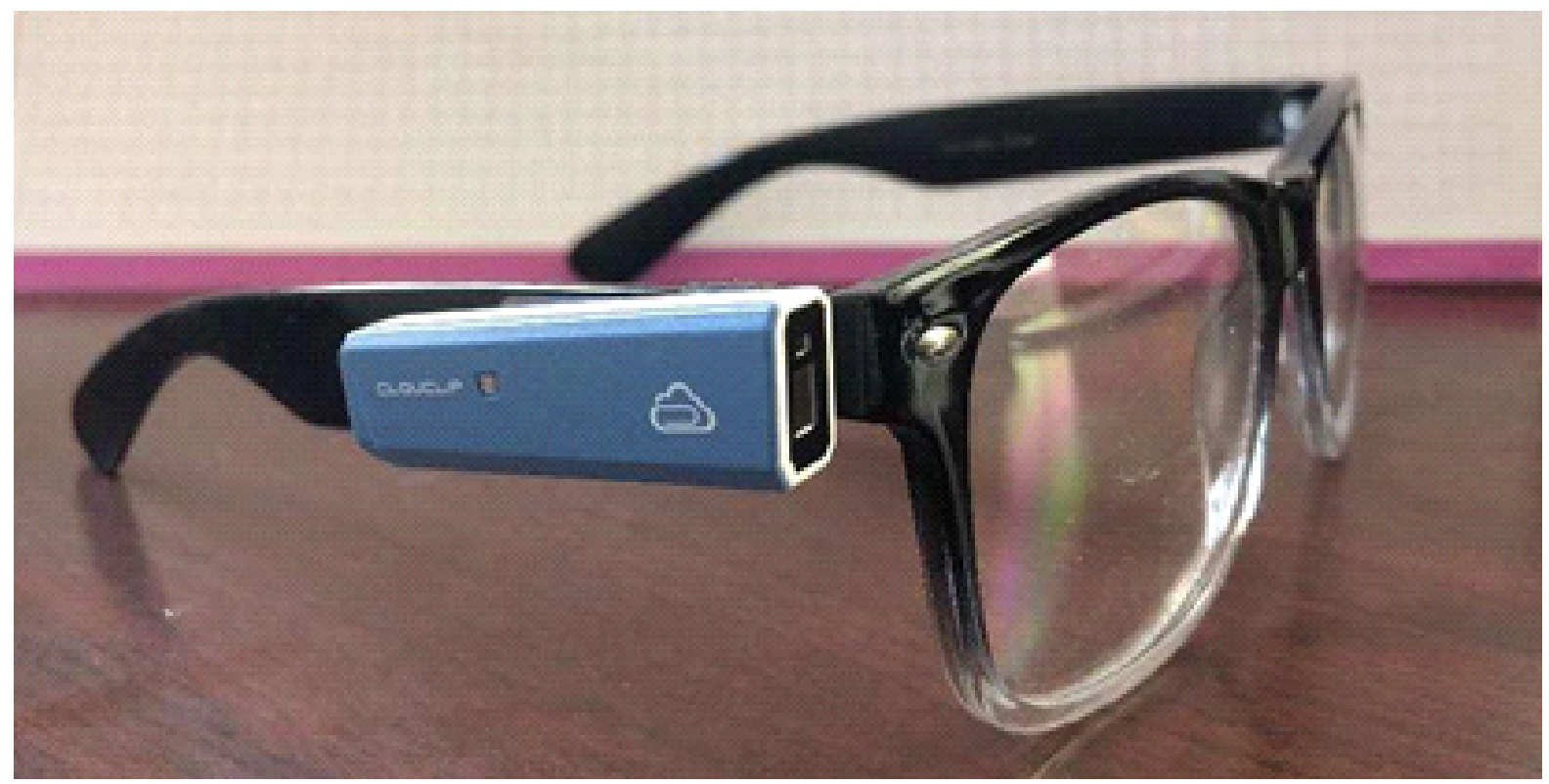

Figure 2. Clouclip attached to front, right temple of Plano glasses

\section{Questionnaire}

Parents were asked to complete the University of Houston Near Work Environmental, and Refractive Error (NEAR) Survey (see supplemental information) regarding demographics and ocular history for themselves and their children. Since environmental factors play a critical role in myopia development (Pan et al., 2012, Eppenberger et al., 2020, Morgan et al., 2012), the questionnaire also inquiries about socioeconomic factors such as the environmental setting and community of where the participants reside and attend school.II

This information provides better insight and context to the background of the sample for the population being studied. Race and ethnicity classification were based on recommendations for the US Census Bureau. Additionally, this survey asks parents to estimate the average number of hours their child spent involved in various activities, both before and during the COVID-19 pandemic. Survey responses were compiled in an Excel spreadsheet where the minimum, maximum, average, and standard deviation for the time spent for each behavior or activity was calculated. Near work was calculated by the total number of hours spent viewing handheld electronic devices, reading printed materials, writing, drawing, painting, and crafting. The total time spent viewing a computer, TV, or handheld electronic devices was used to determine time spend on electronics. Physical activity was defined as hours per day spent in both indoor and outdoor physical activities. Time spent outdoors was determined by adding hours spent riding in a vehicle, as well as in outdoor physical and leisure activities. Equation 1 was used to calculate mean daily hours spent on each activity, and Equation 2 was used to calculate diopter hours, a weighted measure of near work activities.

Equation 1: Mean daily hours $=[($ weekday hours $\times 5)+($ weekend hours $\times 2)] / 7$

Equation 2: Diopter hours $=($ hours near work x $2.8 \mathrm{D})+($ hours intermediate $x 1.6 \mathrm{D})+($ hours far intermediate $\mathrm{x} 0.5 \mathrm{D}$ ) 


\section{Analysis}

Participants were instructed to wear the devices for the entirety of the seven-day measurement period. The Clouclip was worn over the same week that the Actiwatch was worn. All data retrieved from the survey and devices were coded by a participant ID to anonymize the data. No personal information was linked to these participant IDs except for a $\log$ that was stored in a locked cabinet in the lab.

Statistical analysis was performed with SPSS 26 (IBM Corp., Armonk, NY). For questionnaire-derived data, t-tests were conducted to examine differences before and during COVID-19 and between refractive error groups (myopic or non-myopic).

For Actiwatch- and Clouclip-derived data, t-tests between refractive error groups were conducted to compare objective measures of mean daily sleep, activity, light exposure, time outdoors, and near work between refractive error groups.

Data collected from the objective devices, including time outdoors, time spent performing near work, and sleep duration were compared with the data reported in the survey for the same time period using paired t-tests.

\section{Results}

Thirty-one participants, ages $16.3 \pm 0.8$ (mean \pm standard deviation) enrolled in the study. Of these, two participants dropped out for unknown reasons, and there were errors in data collection for nine participants. Data were analyzed for the remaining 20 participants, ages $16.0 \pm 0.8$, including 12 males and 8 females. The refractive error status of the group included 12 myopes and 8 emmetropes.

\section{Demographics}

All participants currently reside in the United States. Four participants were born outside of the United States. Ethnicity was self-reported as Hispanic $(n=3)$ and non-Hispanic $(n=17)$, and racial makeup included Asian $(n=9)$, White $(\mathrm{n}=8)$, African American $(\mathrm{n}=1)$, mixed $(\mathrm{n}=1)$, and unknown $(\mathrm{n}=1)$. Fifteen participants reported living in an urban community and the remaining participants reported living in a suburban community. Eighteen participants reported living in a house and two participants reported living in an apartment. No participants reported living in a townhouse or condominium. While all participants attend school virtually through online learning, two participants were enrolled in a private school and the remaining eighteen were enrolled in a public school.

\section{Subjective Measures}

Questionnaire-derived data are shown in Table 1 and Table 2. Data are shown by session (pandemic versus nonpandemic), day of the week (weekday versus weekend day), and refractive error group (myope vs non-myope). During the pandemic, participants reported spending less time outdoors per day (3.6 \pm 3.1 hours) compared to before the pandemic $(6.6 \pm 4.2$ hours, $\mathrm{P}=.02)$. Physical activity did not vary significantly from before the pandemic $(3.6 \pm 2.6$ hours) compared to during the pandemic $(2.4 \pm 2.2$ hours, $\mathrm{P}=.08)$. Hand-held electronic device use was similar before and during the pandemic $(3.5 \pm 2.5$ and $3.9 \pm 3.0$ hours per day, respectively, $\mathrm{P}=0.3)$, while computer use increased from $4.3 \pm 1.8$ hours per day before the pandemic to $7.1 \pm 3.3$ hours per day during the pandemic $(\mathrm{P}<.001)$. Time spent watching TV did not significantly vary over the pandemic $(\mathrm{P}=0.11)$. There was a significant increase in the amount of overall near and intermediate work from before (10.9 \pm 4.5 hours per day) and during (13.3 \pm 5.1 hours per day) the pandemic $(\mathrm{P}<.001)$, as well as a significant increase in diopter hours from before $(26.0 \pm 11.5)$ to during $(29.8 \pm 12.4$ diopter hours) the pandemic $(\mathrm{P}=.03)$. Participants reported sleeping more per night during the pandemic (7.7 \pm 1.3 hours) compared to before the pandemic $(7.6 \pm 1.6$ hours, $\mathrm{P}=.03)$. 
Table 1. Participant's questionnaire-derived metrics. Mean ((+/-) standard deviation) hours per day spent in outdoor time, physical activity, hand held electronics, computer, TV, and sleep duration for myopic $(\mathrm{n}=13)$ and non-myopic $(n=7)$ teens during a typical school session and during the COVID-19 pandemic on weekdays and weekends.

\begin{tabular}{|l|l|l|l|l|}
\hline & \multicolumn{2}{l|}{ COVID-19 } & \multicolumn{2}{l|}{ PRE COVID-19 } \\
\hline Outdoor Time & Weekday & Weekend & Weekday & Weekend \\
\hline Myopes & $2.4 \pm 2$ & $4.6 \pm 2.5$ & $5.3 \pm 3.9$ & $6.3 \pm 3.8$ \\
\hline Non-Myopes & $4.2 \pm 4.2$ & $5.4 \pm 4.5$ & $5.8 \pm 2.8$ & $7.1 \pm 4.9$ \\
\hline All Participants & $3.1 \pm 3.1$ & $5 \pm 3.4$ & $5.5 \pm 3.4$ & $6.6 \pm 4.2$ \\
\hline Physical Activity & & & & \\
\hline Myopes & $1.9 \pm 1.6$ & $2.3 \pm 1.8$ & $3.4 \pm 3.2$ & $3.3 \pm 2.7$ \\
\hline Non-Myopes & $2.4 \pm 2$ & $3 \pm 2.7$ & $3.4 \pm 1.7$ & $4.5 \pm 1.8$ \\
\hline All Participants & $2.1 \pm 1.7$ & $2.6 \pm 2.2$ & $3.4 \pm 2.7$ & $3.8 \pm 2.4$ \\
\hline Handheld Electronics & & & & \\
\hline Myopes & $3.5 \pm 2.6$ & $3.6 \pm 2.9$ & $2.9 \pm 1.9$ & $4 \pm 2$ \\
\hline Non-Myopes & $4.6 \pm 3.7$ & $4.5 \pm 3.6$ & $4 \pm 2.9$ & $4.1 \pm 3.5$ \\
\hline All Participants & $3.9 \pm 3$ & $4 \pm 3.2$ & $3.4 \pm 2.3$ & $4.1 \pm 2.6$ \\
\hline Computer & & & & \\
\hline Myopes & $7.9 \pm 3.6$ & $6 \pm 3.7$ & $4.2 \pm 1.1$ & $4.9 \pm 2.1$ \\
\hline Non-Myopes & $7.6 \pm 3.7$ & $4.8 \pm 3.1$ & $4 \pm 2.9$ & $5 \pm 3$ \\
\hline All Participants & $7.8 \pm 3.5$ & $5.5 \pm 3.4$ & $4.1 \pm 1.9$ & $5 \pm 2.4$ \\
\hline TV & & & & \\
\hline Myopes & $1.3 \pm 3.2$ & $2.2 \pm 3.4$ & $0.8 \pm 1.3$ & $1.9 \pm 1.8$ \\
\hline Non-Myopes & $0.5 \pm 0.8$ & $1.8 \pm 1$ & $0.5 \pm 0.8$ & $1.6 \pm 1.3$ \\
\hline All Participants & $1 \pm 2.5$ & $2 \pm 2.7$ & $0.7 \pm 1.1$ & $1.8 \pm 1.6$ \\
\hline Sleep Duration & & & & \\
\hline Myopes & $7.3 \pm 1.2$ & $9.2 \pm 1.1$ & $7.2 \pm 1.7$ & $8.8 \pm 1.4$ \\
\hline Non-Myopes & $6.9 \pm 2$ & $9 \pm 1.9$ & $6.9 \pm 2.2$ & $8.8 \pm 1.8$ \\
\hline All Participants & $7.2 \pm 1.5$ & $9.1 \pm 1.4$ & $7.1 \pm 1.9$ & $8.8 \pm 1.5$ \\
\hline
\end{tabular}

Table 2. Participant's questionnaire-derived metrics. Mean ((+/-) standard deviation) hours per day spent on near and intermediate electronics (hand held and computer), all electronics (hand held + computer + TV), near work (hand held + printed + crafts), near/intermediate work (hand held + printed + crafts + computer + board game), for myopic $(\mathrm{n}=$ 13) and non-myopic $(n=7)$ teens during a typical school session and during the COVID-19 pandemic on weekdays and weekends.

\begin{tabular}{|c|c|c|c|c|}
\hline & \multicolumn{2}{|l|}{ COVID-19 } & \multicolumn{2}{|c|}{ PRE COVID-19 } \\
\hline & Weekday & Weekend & Weekday & Weekend \\
\hline \multicolumn{5}{|c|}{ Near and Intermediate Electronics (Handheld and Computer) } \\
\hline Myopes & $11.4 \pm 3.6$ & $9.6 \pm 4.3$ & $7.1 \pm 2.1$ & $8.9 \pm 2.2$ \\
\hline Non-Myopes & $12.2 \pm 4.7$ & $9.3 \pm 6.3$ & $8 \pm 5$ & $9.1 \pm 5.3$ \\
\hline All Participants & $11.7 \pm 4$ & $9.5 \pm 5$ & $7.5 \pm 3.4$ & $9 \pm 3.6$ \\
\hline \multicolumn{5}{|c|}{ All electronics (handheld+ computer + TV) } \\
\hline Myopes & $12.7 \pm 4.9$ & $11.8 \pm 5.3$ & $7.8 \pm 2.7$ & $10.8 \pm 2.7$ \\
\hline
\end{tabular}




\begin{tabular}{|c|c|c|c|c|}
\hline Non-Myopes & $12.7 \pm 5$ & $11 \pm 6.3$ & $8.5 \pm 4.9$ & $10.8 \pm 5.3$ \\
\hline All Participants & $12.7 \pm 4.8$ & $11.5 \pm 5.6$ & $8.1 \pm 3.6$ & $10.8 \pm 3.8$ \\
\hline \multicolumn{5}{|c|}{ Near work (handheld + printed + crafts) } \\
\hline Myopes & $5.2 \pm 3$ & $5.3 \pm 3.4$ & $5.8 \pm 3.3$ & $6.8 \pm 3.7$ \\
\hline Non-Myopes & $7.3 \pm 4.8$ & $7.6 \pm 5.6$ & $7.1 \pm 3.7$ & $6.8 \pm 4.9$ \\
\hline All Participants & $6 \pm 3.9$ & $6.2 \pm 4.4$ & $6.3 \pm 3.4$ & $6.8 \pm 4.1$ \\
\hline \multicolumn{5}{|c|}{ Near/Intermediate work (handheld + printed + crafts + computer + board game $)$} \\
\hline Myopes & $13.1 \pm 3.9$ & $11.4 \pm 4.5$ & $10 \pm 3.4$ & $12.2 \pm 5$ \\
\hline Non-Myopes & $15.1 \pm 6.1$ & $12.9 \pm 8.5$ & $11.3 \pm 5.9$ & $11.9 \pm 6.3$ \\
\hline All Participants & $13.9 \pm 4.9$ & $12 \pm 6.2$ & $10.5 \pm 4.5$ & $12.1 \pm 5.4$ \\
\hline
\end{tabular}

The duration of hand-held Electronics $(\mathrm{P}=0.45)$, time spent on computer $(\mathrm{P}=0.72)$, television $(\mathrm{P}=0.55)$, near and intermediate electronics $(\mathrm{P}=0.80)$, all electronics $(\mathrm{P}=0.92)$, near work $(\mathrm{P}=0.23)$, or sleep $(\mathrm{P}=0.60)$ did not vary significantly by refractive error.

\section{Objective Measures}

Actiwatch-derived data showed that over one week during the pandemic, participants experienced an average daily light exposure of $198 \pm 194$ lux and spent $32 \pm 27$ minutes outdoors (exposed to $>1000$ lux) per day. Participants slept $8.3 \pm 1.2$ hours per night. There were no differences between refractive error groups for daily light exposure $(\mathrm{P}$ $=.89)$, time outdoors $(\mathrm{P}=.44)$, or sleep duration $(\mathrm{P}=.80)$.

A

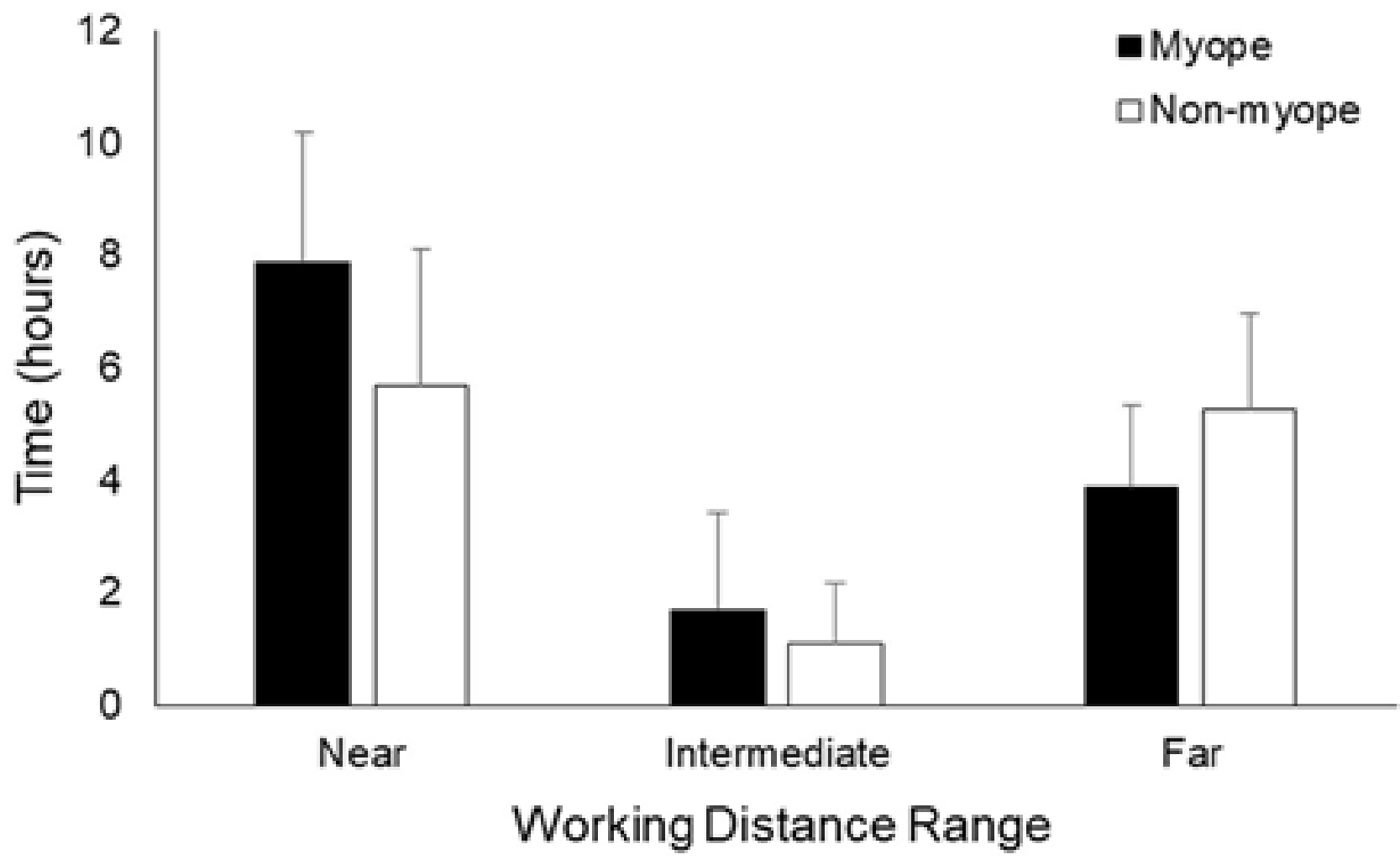


B

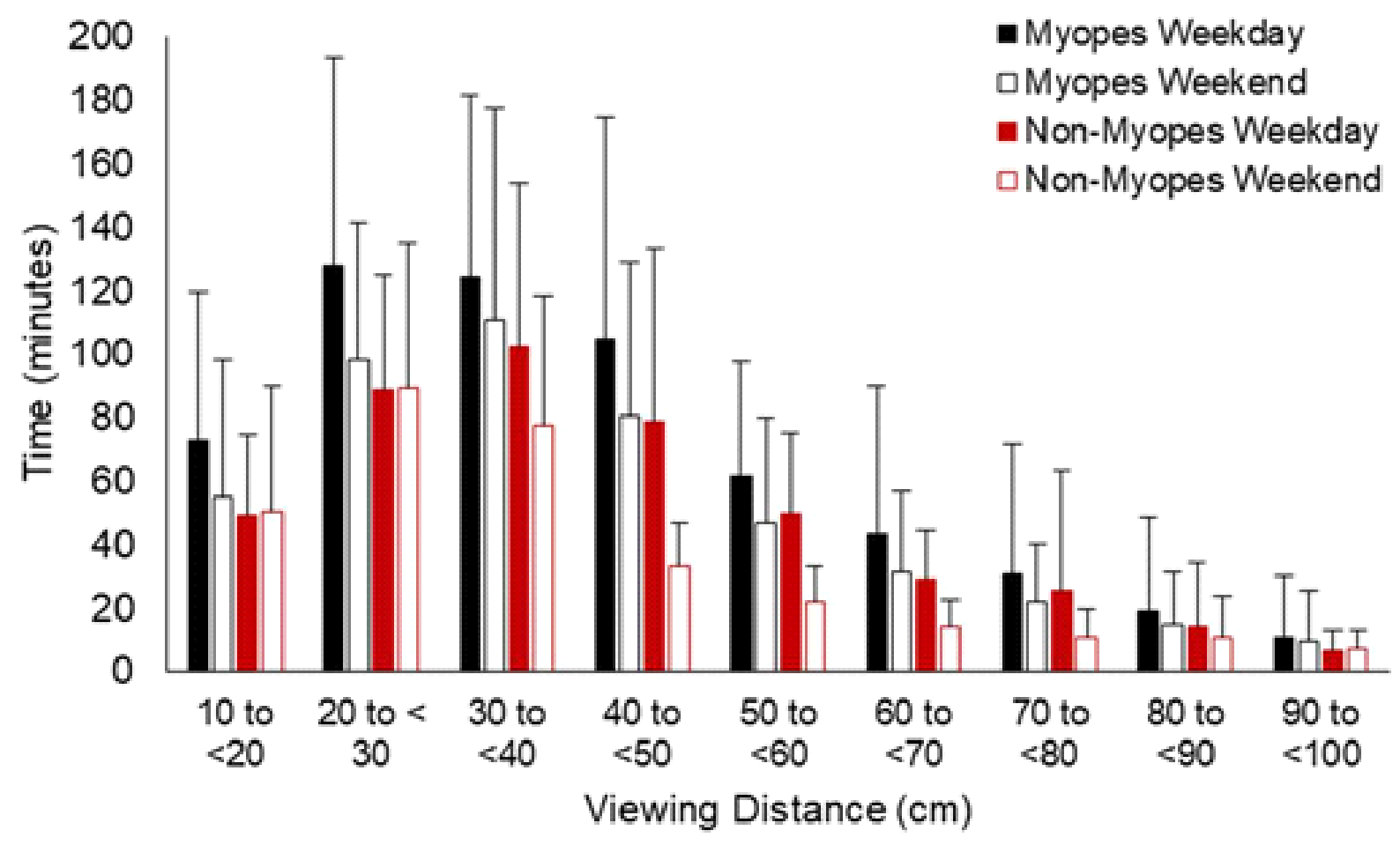

Figure 1: A) Clouclip-measured mean hours per day spent in near work, intermediate work, and far work for myopes (solid bars) and non-myopes (open bars). B) Clouclip-measured mean minutes per day spent in various working distance bins on weekdays (solid bars) and weekends (open bars) for myopes (black) and non-myopes (red). Near work is defined as a working distance of $10-60 \mathrm{~cm}$, while intermediate work is about $60-100 \mathrm{~cm}$, and far work is defined as a working distance greater than $100 \mathrm{~cm}$.

Participants wore the Clouclip for $6.9 \pm 0.8$ days with a total wear time of $14.6 \pm 1.0$ hours per day. Working distance data are shown by day of the week and refractive error group in $10 \mathrm{~cm}$ bins in Figure 1. Clouclip-derived data showed that myopic participants engaged in near work (working distances of 10 to $<60 \mathrm{~cm}$ ) for $8.4 \pm 2.6$ hours on a weekday and $6.5 \pm 2.1$ hours on a weekend day. Non-myopic participants engaged in near work for $6.1 \pm 2.7$ hours on a weekday and $4.5 \pm 1.9$ hours on a weekend day. While weekend near work was significantly different between refractive error groups $(\mathrm{P}<.05)$, weekday near work $(\mathrm{P}=.08)$ was not.

\section{Discussion}

Objectively measured data from the Clouclip indicated that myopes consistently performed near work for greater durations of time than non-myopes. On the other hand, questionnaire-derived subjective results indicated that behaviors were not associated with myopia. These findings highlight the need for incorporating objective measures, rather than subjective questionnaires, to more precisely quantify the contributions of behaviors to myopia. Over the pandemic, there was no significant change in sleep duration and physical activity in the general population of Houston high schoolers regardless of refractive error, while results have revealed a significant decrease in outdoor light exposure and the increase in near and intermediate work, especially with electronic devices such as the computer. This increase in electronic device use and minimized outdoor light exposure may be a result of the mandatory requirements 
posed by high schools. Students must attend classes online and complete a set amount of homework online; these mandatory durations of screen time do not vary by refractive error since all students have the same requirements. As the Spring semester of 2021 concludes and many students are transitioning back into in-person learning, durations of these behaviors are bound to change. While behaviors did not significantly vary by refractive error group on weekdays due to online schooling, there was a significant difference in behaviors during the weekend where there were no mandatory screen-time hours. A study conducted over the summer where individuals may have more freedom in their behaviors may yield different results.

\section{Limitations and Assumptions}

One limitation in the current study was subject compliance and reliable data. Data were excluded for days that participants removed the device for a period greater than 30 minutes, or days that a light exposure of zero was recorded for an interval greater than 30 minutes, indicating that the light sensor was obstructed by clothing. A limitation of a crosssectional study is that the data is collected from a specific point in time and is not longitudinal; therefore, data was not measured over a period of time over the pandemic, hence the need for self-reported data for behaviors before the pandemic.

Therefore, another drawback of this study was the reliance on self-reported data. Selfreported data is prone to social desirability bias and may lead to less reliable data and erroneous conclusions (Latkin et al., 2018). Additionally, rating scale questions, such as the ones administered in the UH NEAR survey, are also subjective and may be biased. However, due to its less time-consuming and convenient nature, rating scale questions have become a standard for collecting quantitative and qualitative data in the field of research.

Limitations in the current study include the small sample size. An increased sample size utilizing objective measures of near work, sleep, and light exposure may strengthen the trends in behavioral patterns observed between refractive error groups (Ostrin et al., 2019). These 20 students who participated in the study represented 6 different high schools. 11 of these students attend a vanguard-only high school, which may bias the amount of homework and screen time these students took part in.

In this study, it is assumed that the data collected in this one-week time frame is representative of the individual's average behavior. It is also assumed that wearing the Clouclip during the day does not influence the participant's daily activities, and that wearing the watch to sleep does not affect the participant's sleeping pattern and behaviors. It is also assumed that the amount of time participating in each activity reported by the participant is an accurate estimation of their behaviors.

\section{Future Research}

With the pandemic being so recent, future studies could help by researching the impact of the coronavirus on the biology of the eye, if any, and its impact on vision. These findings may be measured in additional studies to identify its effect on myopia, if any. Since all participants in this study were enrolled in online-school, this study is unable to identify any comparisons between those enrolled in in-person school and those schooling virtually. Studies including participants who are homeschooled or have transitioned back into in-person learning may be beneficial to the field as it would highlight eh differences in behaviors of those online and inperson during the pandemic. Myopia prevalence varies by geographic location (Eppenberger et al., 2020, Morgan et al., 2012). Environmental factors play a critical role in myopia development (Pan et al., 2012) from a young age as refractive error can vary depending on if children live in rural or urban environments (Gao et al., 2012). This same study conducted in different geographic locations with a more diverse subject population would enhance the understanding of the impact of the pandemic on students. Future studies could include participants from urban, suburban, and rural areas and have a more ethnically broader population subset. 


\section{Conclusion}

Ultimately, this study was able to accomplish its purpose of identifying behaviors associated with the pandemic and how they compared between myopes and non-myopes. My results do not fully align with my hypothesis. My hypothesis aligns with my results in the sense that there has been a change in behavior over the course of the pandemic, however, my results contradict my hypothesis as they indicate that behaviors do not significantly vary by refractive error. Though the idea that behaviors have changed over the pandemic could have been assumed earlier, there was previously no statistics or evidence to support that claim. These results are significant because this new understanding of the change in behaviors over the pandemic may allow professionals to provide more accurate behavioral recommendations to mitigate the risk of myopia. These results may also be of interest to those studying child behavior, public health, myopia, or even just the influence of the pandemic.

\section{Acknowledgments}

This work is ostensibly supported by the Ostrin Lab at the University of Houston College of Optometry. Approval from the University of Houston Institutional Review Board for STUDY00002637 is appreciated. Special thank you to my mentor, Dr. Lisa Ostrin, for assistance with device availability and guidance with the research process.

\section{References}

Bélanger, M. È., Bernier, A., Paquet, J., Simard, V., \& Carrier, J. (2013). Validating actigraphy as a measure of sleep for preschool children. Journal of clinical sleep medicine : JCSM : official publication of the American Academy of Sleep Medicine, 9(7), 701-706. https://doi.org/10.5664/jcsm.2844

Bhandari, K.R., \& Ostrin, L.A. (2020).Validation of the Clouclip and utility in measuring viewing distance in adults. Ophthalmic Physiol Opt 2020; 40: 801- 814. https://doi.org/10.1111/opo.12735

Chia, KS; Hong, CY; Tan, D; Saw, SM; Stone, RA; (2001) Nearwork and Myopia in Young Children The Lancet https://doi.org/10.1016/S0140-6736(05)71520-8

Eppenberger, L. S., \& Sturm, V. (2020). The Role of Time Exposed to Outdoor Light for Myopia Prevalence and Progression: A Literature Review. Clinical ophthalmology (Auckland, N.Z.), 14, 18751890. https://doi.org/10.2147/OPTH.S245192

Flitcroft D. I. (2012). The complex interactions of retinal, optical and environmental factors in myopia aetiology. Progress in retinal and eye research, 31(6), 622-660.

https://doi.org/10.1016/j.preteyeres.2012.06.004

Gao, Z., Meng, N., Muecke, J., Chan, W. O., Piseth, H., Kong, A., Jnguyenphamhh, T., Dehghan, Y., Selva, D., Casson, R., \& Ang, K. (2012). Refractive error in school children in an urban and rural setting in Cambodia. Ophthalmic epidemiology, 19(1), 16-22. https://pubmed.ncbi.nlm.nih.gov/22273355/

Gill, B. P., Schlossman, S. L. (2010). Villain or Savior? The American Discourse on Homework, 1850-2003, Theory into Practice, 43:3, 174-181. https://doi.org/10.1207/s15430421tip4303 2 
Harb, E., Thorn, F., \& Troilo, D. (2006). Characteristics of accommodative behavior during sustained reading in emmetropes and myopes. Vision research, 46(16), 2581-2592. https://doi.org/10.1016/j.visres.2006.02.006

Holden, BA; Fricke, TR; Wilson, DA; Jong, M; Naldoo, KS; Sankaridurg, P; Wong, TY; Naduvilath, TJ (2016) Global Prevalence of Myopia and High Myopia Temporal Trends from 2000 through 2050. American Academy of Ophthalmology https://doi.org/10.1016/j.ophtha.2016.01.006

Holshue, M. L., DeBolt, C., Lindquist, S., Lofy, K. H., Wiesman, J., Bruce, H., Spitters, C., Ericson, K., Wilkerson, S., Tural, A., Diaz, G., Cohn, A., Fox, L., Patel, A., Gerber, S. I., Kim, L., Tong, S., Lu, X., Lindstrom, S., Pallansch, M. A., ... Washington State 2019-nCoV Case Investigation Team (2020). First Case of 2019 Novel Coronavirus in the United States. The New England journal of medicine, 382(10), 929-936. https://doi.org/10.1056/NEJMoa2001191

Huang, H. M., Chang, D. S., \& Wu, P. C. (2015). The Association between Near Work Activities and Myopia in Children-A Systematic Review and Meta-Analysis. PloS one, 10(10), e0140419. https://doi.org/10.1371/journal.pone.0140419

Jee, D., Morgan, I. G., \& Kim, E. C. (2016). Inverse relationship between sleep duration and myopia. Acta ophthalmologica, 94(3), e204-e210. https://doi.org/10.1111/aos.12776

Landis, E. G., Yang, V., Brown, D. M., Pardue, M. T., \& Read, S. A. (2018). Dim Light Exposure and Myopia in Children. Investigative ophthalmology \& visual science, 59(12), 4804-4811. https://doi.org/10.1167/iovs.18-24415

Lanca, C \& Saw, S-M. (2020) The association between digital screen time and myopia: A systematic review. Ophthalmic Physiol Opt 2020; 40: 216- 229. https://doi.org/10.1111/opo.12657

Latkin, C. A., Edwards, C., Davey-Rothwell, M. A., \& Tobin, K. E. (2017). The relationship between social desirability bias and self-reports of health, substance use, and social network factors among urban substance users in Baltimore, Maryland. Addictive behaviors, 73, 133-136. https://doi.org/10.1016/j.addbeh.2017.05.005

Lougheed T. (2014). Myopia: the evidence for environmental factors. Environmental health perspectives, 122(1), A12-A19. https://doi.org/10.1289/ehp.122-A12

Lu, B., Congdon, N., Liu, X., Choi, K., Lam, D. S., Zhang, M., Zheng, M., Zhou, Z., Li, L., Liu, X., Sharma, A., \& Song, Y. (2009). Associations between near work, outdoor activity, and myopia among adolescent students in rural China: the Xichang Pediatric Refractive Error Study report no. 2. Archives of ophthalmology (Chicago, Ill. : 1960), 127(6), 769-775. https://doi.org/10.1001/archophthalmol.2009.105

Morgan, I. G., Ohno-Matsui, K., \& Saw, S. M. (2012). Myopia. Lancet (London, England), 379(9827), 1739-1748. https://doi.org/10.1016/S0140-6736(12)60272-4

Navel, V., Beze, S., \& Dutheil, F. (2020). COVID-19, sweat, tears... and myopia?. Clinical \& experimental optometry, 103(4), 555. https://doi.org/10.1111/cxo.13086

Ostrin, L. A., Sajjadi, A., \& Benoit, J. S. (2018). Objectively Measured Light Exposure During School and Summer in Children. Optometry and vision science : official publication of the American Academy of Optometry, 95(4), 332-342. https://doi.org/10.1097/OPX.0000000000001208 
Pan, C. W., Ramamurthy, D., \& Saw, S. M. (2012). Worldwide prevalence and risk factors for myopia. Ophthalmic \& physiological optics: the journal of the British College of Ophthalmic Opticians (Optometrists), 32(1), 3-16. https://doi.org/10.1111/j.1475-1313.2011.00884.x

Read, S. A., Collins, M. J., \& Vincent, S. J. (2014). Light exposure and physical activity in myopic and emmetropic children. Optometry and vision science : official publication of the American Academy of Optometry, 91(3), 330-341. https://doi.org/10.1097/OPX.0000000000000160

Saw, S. M., Nieto, F. J., Katz, J., Schein, O. D., Levy, B., \& Chew, S. J. (2000). Factors related to the progression of myopia in Singaporean children. Optometry and vision science : official publication of the American Academy of Optometry, 77(10), 549-554. https://doi.org/10.1097/00006324-200010000-00009

Smith, M. J., \& Walline, J. J. (2015). Controlling myopia progression in children and adolescents. Adolescent health, medicine and therapeutics, 6, 133-140. https://doi.org/10.2147/AHMT.S55834

Theophanous, C., Modjtahedi, B. S., Batech, M., Marlin, D. S., Luong, T. Q., \& Fong, D. S. (2018). Myopia prevalence and risk factors in children. Clinical ophthalmology (Auckland, N.Z.), 12, 15811587. https://www.ncbi.nlm.nih.gov/pmc/articles/PMC6120514/

Velavan, T. P., \& Meyer, C. G. (2020). The COVID-19 epidemic. Tropical medicine \& international health : TM \& IH, 25(3), 278-280. https://doi.org/10.1111/tmi.13383

Wen, L., Cao, Y., Cheng, Q., Li, X., Pan, L., Li, L., Zhu, H., Lan, W., \& Yang, Z. (2020). Objectively measured near work, outdoor exposure and myopia in children. The British journal of ophthalmology, 104(11), 1542-1547. https://doi.org/10.1136/bjophthalmol-2019-315258 